\title{
Oblique tibial osteotomy with elevation of medial tibial plateau for treatment of tibia vara (Blount's disease) in 8 year old girl: (case report)
}

\begin{abstract}
Blount's disease is a progressive form of genu varum due to asymmetrical inhibition of the medial portion of the proximal tibial epiphysis. The surgical treatments involved in correction of Blount's disease are often demanding. In 1988 George T. Rab developed a new method for surgical correction of the tibia vara (Blount's disease) via Oblique proximal tibial osteotomy which corrects it in two planes with excellent cosmetic appearance, in severe cases associated with depression; elevation osteotomy of the medial tibial plateau may be added. This technique will be described in an 8-year-old girl who presented with bilateral tibia vara (blount disease) with excellent result at 6 months of follow up.
\end{abstract}

Case Report

Volume 10 Issue I - 2018

\author{
Ghazi Fannouch, Sami Bin Dagher, Saleh \\ Alfaisali \\ King Saud Bin Abdulaziz University for Health Science (KSAU \\ $\mathrm{HS})$, King Abdullah Specialized Children Hospital (KASCH), \\ Saudi Arabia

\begin{abstract}
Correspondence: Saleh Alfaisali, King Saud Bin Abdulaziz University for Health Science (KSAUHS), King Abdullah Specialized Children Hospital (KASCH), Ministry of National Guard -Health Affairs, PO Box 22490, Riyadh I I 426, Saudi Arabia,Email dr.alfaisali@gmail.com
\end{abstract}

Received: February 18, 2018 | Published: February 28, 2018

\section{Introduction}

Tibia vara Blount's disease is a developmental condition characterized by disordered endochondrial ossification of the medial part of the proximal tibial physis. In this condition which was first described by Erlancher in 1922 and later reviewed by Blount in 1937, ${ }^{1}$ there is a complex three-dimensional deformity, which typically includes varus, internal rotation, and possible procurvatum. ${ }^{2}$ It results from repeated compressive loading injury of proximal medial tibial physis with overgrowth of lateral tibial physis leading to varus deformity. ${ }^{3}$

Surgical correction of Blount disease can be achieved by number of methods, which includes asymmetric temporary physeal stapling, hemiepiphysiodesis, or osteotomy. ${ }^{4,5}$

In 1988 George T .Rab developed a new method for correction of the tibia vara (Blount's disease) via Oblique proximal tibial osteotomy. The main advantages of this technique are the maintenance of significant cancellous bone contact while correcting deformities in two planes, and the excellent cosmetic appearance afforded by both the bi-planar correction. ${ }^{6}$

Storn et al. ${ }^{7}$ in 1970 were the first to describe a hemi condylar tibial osteotomy through the epiphysis into the intercondylar notch. This method correct the medial slope of the tibial epiphysis while avoiding the physis. ${ }^{7-9}$

We present our experience with eight year old girl with blount's disease whom we treated with double proximal tibial osteotomy, All the figures in this case report are exclusive and original.

\section{Case report}

An eight -year-old girl presented to us with progressive bowing of both legs for the last 4 years causing difficulty in walking. She did not have any history of trauma or fever. There was no family history of any leg deformities. On clinical examination we found internal tibial torsion and bilateral proximal tibial varus. She had full painless range of motion in both hips and knees, without any limb length discrepancy. Radiographs revealed bilateral depression of medial cortical wall of the proximal tibial metaphysis, metaphyseal diaphyseal angle is 13 degrees on the right side and 25 degrees on the left side (Figure 1AC).
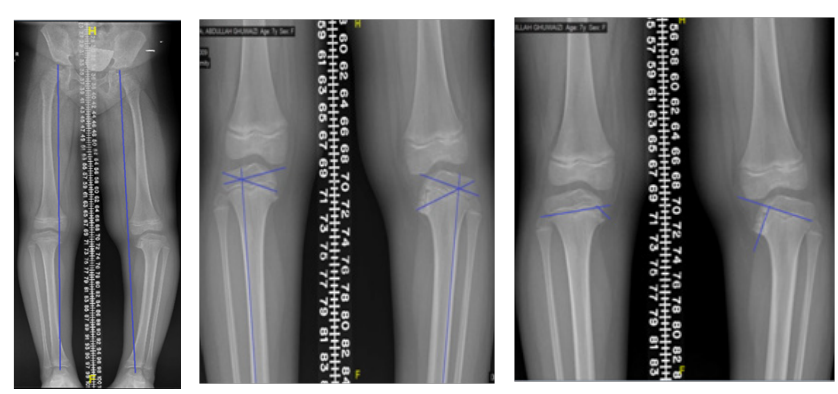

Figure I 8 years old with blount disease:A. mechanical axis, B metaphyseal diaphyseal angle, $\mathrm{C}$ medial physeal slope.

Routine hematological investigations were normal. Based on the above investigations, diagnosis of Blount's disease was made and classified according to Langenskiöld classification (1952). As stage II on the right side and stage IV on the left side.

\section{Operative technique:(double proximal tibial ostetomy)}

In supine position on a radiolucent table, an osteotomy of the fibula was performed at the junction of the proximal and middle thirds through $4-\mathrm{cm}$ incision. About $1 \mathrm{~cm}$ of fibula was excised. Through a transverse incision at the lower pole of the tibial tubercle, 
the periosteum was incised in Y-shaped fashion, and dissected subperiosteally. The anteromedial and anterolateral surfaces of the proximal tibial metaphysis and diaphysis were subperiosteally exposed. Hohmann's retractors were introduced subperiosteally with the knee in flexion, to prevent injury to the neurovascular structures. Under image intensifier control, a smooth $\mathrm{K}$-wire was drilled into the tibia at the angle of 45 degrees. The pin was inserted $1 \mathrm{~cm}$ distal to the proximal tibial tubercle and directed toward the posterior cortex to terminate at a safe distance from the growth plate., we started the plane to correspond with the distal, internally rotated tibial surface, with power saw the tibia was osteotomized distal to the pin, the osteotomized tibia was manipulated, correcting both varus and medial rotation. Fixation of the fragment was done by a screw. The desire angle was checked by drawing imaginary line of mechanical axis using the diathermy lead, then a $2^{\text {nd }}$ screw was inserted and engaged to the posterior tibial cortex..$^{10,11}$

With extension of the same incision medially, the medial tibial plateau was exposed extraperiosteally, to avoid any periosteal damage. Using an image intensifier, a guide-wire was placed transversely, parallel to the sloping condyle as closely as possible, to the tibial plateau to the midline under tibial eminence. The sloping condyle was elevated with a wide osteotomy and distracted using lamina spreader. Wedge-shaped bone allografts were inserted under the fragment with maximal forced valgus of the knee.

The position of the osteotomy was verified by image intensifier. The tourniquet was released and complete hemostasis was achieved. Fasciotomy of the anterior compartment was performed to prevent compartmental syndrome. A plaster cast was applied under forced valgus position (Figure 2).
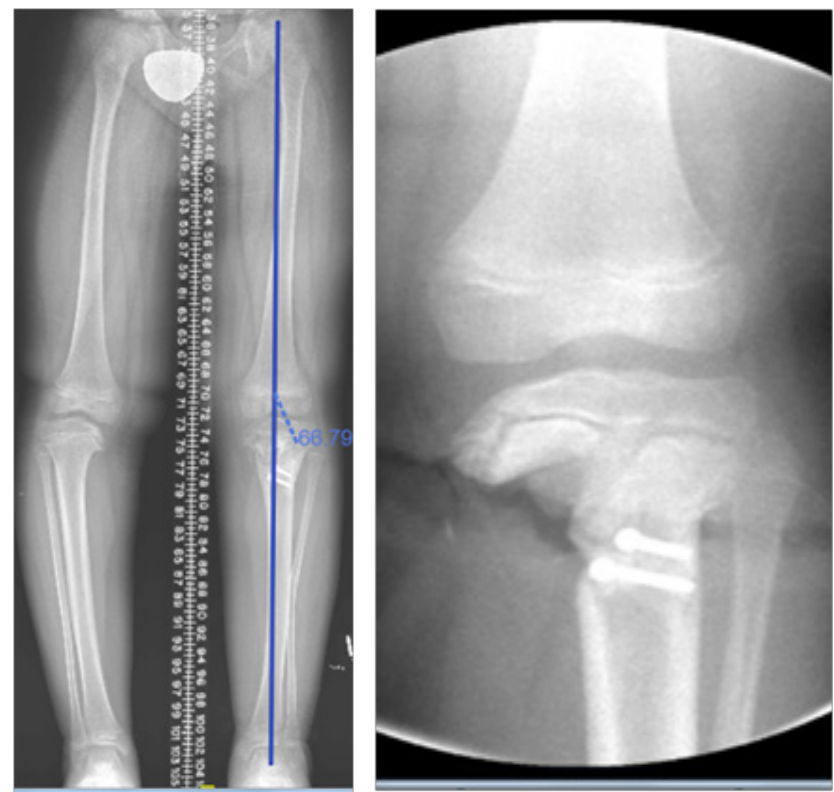

Figure 2 post operation: A mechanicl axis, B osteotomy site post correction.

Eight weeks later temporary hemi-epiphysiodesis was done for right lateral proximal tibia using (Orthofix) 8-plate to correct the deformity of the right tibia. The patient was allowed weight bearing after that (Figure 3).
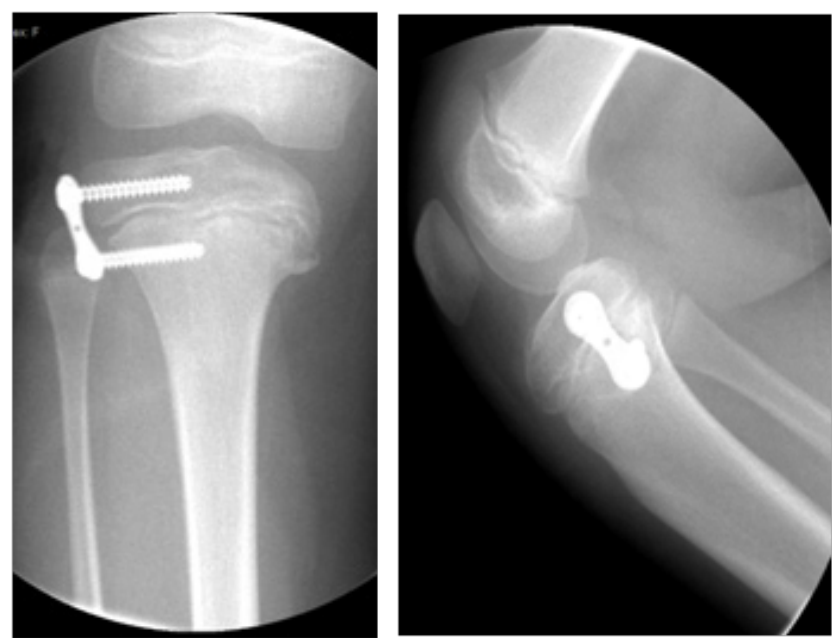

Figure 3 (A \& B) AP, LAT views of the right tibial post temporary hemiepiphysiodesis.

Later, patient was seen in the clinic with free of pain and full range of motion, $\mathrm{x}$-ray showed good healing of the osteotomies (Figure 4AF).
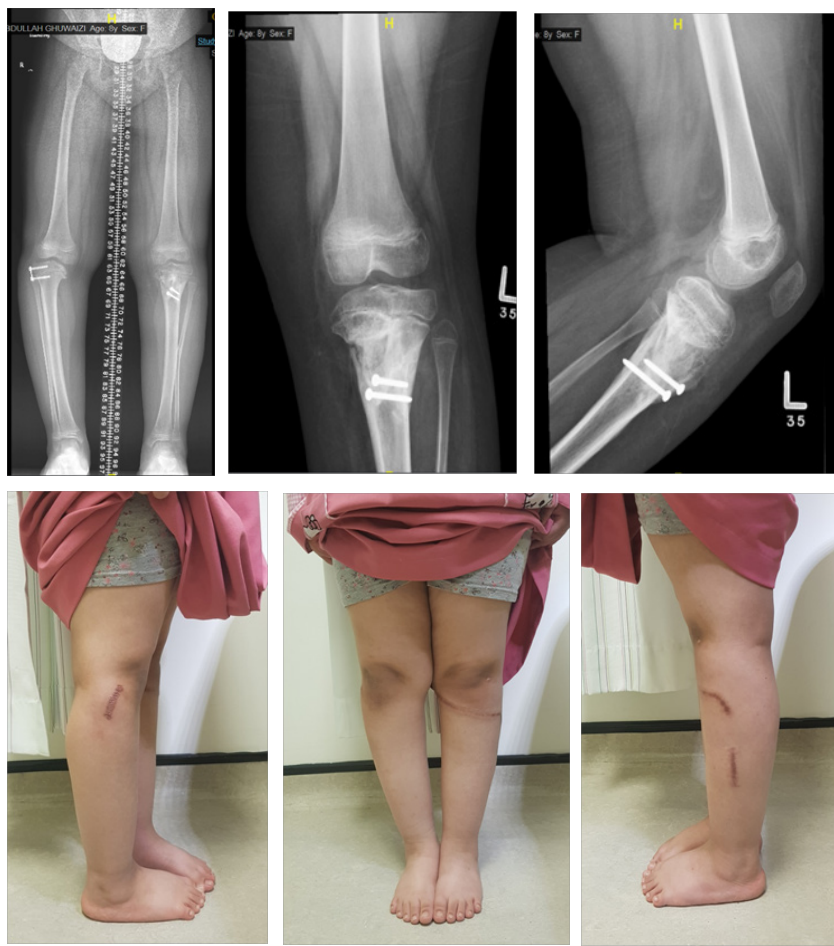

Figure 4 (A-F) 6 months post operation.

\section{Discussion}

The best way to obtain surgical correction of tibial deformity should be safe, provide reliable correction with minimum morbidity and be reproducible. Oblique tibial osteotomy corrects accurately both the angular and rotational mal-alignment whilst maintaining bone length. Good bony apposition occurred in our case. With the 
added hemicondylar tibial osteotomy of lateral cortex after rotation of the distal fragment, excellent bone elevation was achieved. The limited internal fixation with crossed screws provided satisfactory control of the osteotomy position. ${ }^{12}$

Elzohairy et al. ${ }^{2}$ described 17 patients with tibia vara. A total of 29 tibiae underwent corrections of the deformity associated with tibia vara by modified Rab proximal tibial oblique osteotomy. They modified the procedure by using k.wires for fixation and changing the direction of the osteotomy. According to Schoenecker's criteria, $100 \%$ of the patients in this study had a good result. ${ }^{2}$

Zayer et al. ${ }^{8}$ published a case report of two neglected cases of Blount's disease with severe sloping of the medial tibial condyle and excessive ligamentous laxity. They did elevation of the medial tibial condyle with good results. ${ }^{8}$

\section{Conclusion}

The Rab oblique proximal tibial osteotomy is a useful technique and has advantage of allowing both angular and rotational deformities correction while maintain length, stability and metaphyseal contact. In severe cases associated with depression, elevation osteotomy of the medial tibial plateau (double tibial osteotomy technique) is needed to restore the joint architecture.

\section{Acknowledgements}

None.

\section{Conflict of interest}

Authors declare there is no conflict of interest in publishing the article.

\section{References}

1. Blount WP. Tibia Vara: Osteochondrosis deformans tibiae. J Bone Joint Surg Am. 1937;19:1-29.

2. Elzohairy MM. Modified oblique proximal tibial osteotomy for deformity correction of tibia vara (blount disease). Orthop Muscul Syst. 2012; $1: 118$.

3. Langenskiöld A. Tibia vara (osteochondrosis deformans tibiae); a survey of 23 cases. Acta Chir Scand. 1952;103(1):1-22.

4. Franck Accadbled. One-step treatment for evolved blount's disease, four cases and review of the literature. J Pediatr Orthop. 2003;23(6):747-52.

5. Samir Sabharwal, Sanjeev Sabharwal. Treatment of infantile blount disease: an update. J Pediatr Orthop. 2017;37 Suppl 2:S26-S31.

6. Rab GT. Oblique tibial osteotomy for blount's disease (tibia vara). J Pediatr Orthop. 1988;8:715-720.

7. Støren H. Operative elevation of the medial tibial joint surface in Blount's disease. Acta Orthop Scand. 1970;40:788.

8. Zayer M. Hemicondylar tibial osteotomy in blount's disease: a report of two cases. Acta Orthop Scand. 1970;63:350.

9. Langenskiold A. Tibia vara; (osteochondrosis deformans tibiae); a survey of 23 cases. Acta Chir Scand. 1952;103(1):1-22.

10. Raju Karuppal. Case report: 'Z' osteotomy - a novel technique of treatment in blount's disease. F1000Research. 2015;4:1250.

11. Rab GT. Oblique tibial osteotomy revisited. $J$ Child Orthop. 2010;4(2):169-72.

12. Schoenecker PL, Johnston R, Rich MM, et al. Elevation of the medial plateau of the tibia in the treatment of blount disease. J Bone Joint Surg. 1992;74A:351. 